\title{
Renormalization of Quantum Anosov Maps: Reduction to Fixed Boundary Conditions
}

\author{
Itzhack Dana \\ Minerva Center and Department of Physics, Bar-Ilan University, Ramat-Gan 52900, Israel
}

\begin{abstract}
A renormalization scheme is introduced to study quantum Anosov maps (QAMs) on a torus for general boundary conditions (BCs), whose number $(k)$ is always finite. It is shown that the quasienergy eigenvalue problem of a QAM for all $k$ BCs is exactly equivalent to that of the renormalized QAM (with Planck's constant $\hbar^{\prime}=\hbar / k$ ) at some fixed BCs that can be of four types. The quantum cat maps are, up to time reversal, fixed points of the renormalization transformation. Several results at fixed BCs, in particular the existence of a complete basis of "crystalline" eigenstates in a classical limit, can then be derived and understood in a simple and transparent way in the general-BCs framework.
\end{abstract}

PACS numbers: 05.45.Mt, 03.65.Ca, 03.65.Sq, 11.10.Gh

Typeset using REVTEX 
Nonintegrable systems whose dynamics can be reduced to a $2 \mathrm{D}$ torus in phase space have attracted much attention in the quantum-chaos literature. When quantizing such a system on the torus, the admissible quantum states must satisfy proper boundary conditions (BCs), i.e., they have to be periodic on the torus up to constant phase factors specified by a Bloch wave vector $\mathbf{w}$. If the Hamiltonian of the system is periodic in phase space, e.g., the kicked Harper model [1 4, its classical dynamics can be reduced to the toral phase space of one unit cell of periodicity, and all Bloch wave vectors w in some Brillouin zone (BZ) are allowed. The sensitivity of the eigenstates to continuous variation of $\mathbf{w}$ in the BZ may be characterized topologically by a Chern index 표 and appears to be strong for eigenstates spread over the chaotic region and weak for eigenstates localized on stability islands.

In general, however, the Hamiltonian of a system whose dynamics can be reduced to a torus is not periodic in phase space. An extreme case is that of the purely chaotic, Anosov "cat maps" [5-14], whose Hamiltonians are quadratic in the phase-space variables [9]. When quantizing these systems, it turns out that only a finite set of w's in the BZ is allowed [10 15], see Eq. (2) below, and this set increases with increasing chaotic instability. However, due to the lack of $2 \mathrm{D}$ continuity in this set, the sensitivity to variations in $\mathbf{w}$ cannot be characterized by a Chern index [15]. A large class of cat maps was first quantized by Hannay and Berry [6] for $\mathbf{w}=\mathbf{0}$ (strict periodicity on the torus) and general BCs were considered in subsequent works [10 15]. An important recent work [14] has shown the existence of eigenstates whose Husimi density is periodic on a lattice in the torus and whose Husimi zeros form a crystal, for all Planck's constants in a sequence tending to zero. These "crystalline" eigenstates are the first example of exactly equidistributed eigenstates of quantized chaotic systems in a classical limit.

An atypical feature of quantum cat maps is their high spectral degeneracy, which increases in the classical limit [6,9]. Typical spectral properties, fitting generic eigenvalue statistics, are already found by quantizing torus maps that are very slight perturbations of 
the cat maps [16 [18]. According to Anosov's theorem [5], these maps have essentially the same classical dynamics, in particular they are purely chaotic, as the unperturbed cat maps, which are structurally stable. This ceases to be the case for larger perturbations that cause bifurcations generating elliptic islands and thus a mixed phase space [18]. However, the quantum BCs for a perturbed cat map are the same as those of the unperturbed cat map, independently of the size of the perturbation [15], see Eq. (2) below. Because of this reason and to simplify terms, general perturbed cat maps are referred to as Anosov maps in this Letter. The importance of these maps is in that they may be viewed as generic torus maps on the basis of a general expression for a smooth torus map derived recently [15, see below.

In this Letter, we introduce a renormalization scheme to study quantum Anosov maps (QAMs) for general BCs. We show that the eigenvalue problem of a QAM for all BCs, determining the full quantum dynamics, can be exactly reduced to a problem at fixed BCs. More specifically, consider a QAM given by the evolution operator $\hat{U}$ quantizing a classical Anosov map. Quantization on a torus requires a Planck's constant $\hbar$ to satisfy $2 \pi / \hbar=p$, an integer. The finite number of BCs is denoted by $k$, which depends only on the classical unperturbed cat map. We define a renormalization transformation $\mathcal{R}$ of $\hat{U}$ generating a QAM $\hat{U}^{\prime}=\mathcal{R}(\hat{U})$ on the same torus. The number of BCs for $\hat{U}^{\prime}$ is also $k$ and $\hat{U}^{\prime}$ is associated with a renormalized Planck's constant $\hbar^{\prime}=\hbar / k$. We then show that the quasienergy eigenvalue problem for $\hat{U}$ for all $k$ BCs is equivalent, by a unitary transformation accompanied by a scaling of variables, to that for $\hat{U}^{\prime}$ at some fixed BCs. The latter can be of four types, corresponding to the four "symmetry" points in the renormalized BZ (see Table 1 below). The quantum cat maps are always fixed points of $\mathcal{R}$ in the reflection-hyperbolic case. In the ordinary-hyperbolic case, they are fixed points of $\mathcal{R}$ only if some conditions are satisfied. Otherwise, they are fixed points of $\mathcal{R}$ accompanied by time reversal. Thus, for general $\hat{U}$, the QAMs $\hat{U}^{(n)}=\mathcal{R}^{n}(\hat{U})$ represent perturbations of a given quantum cat map, or its time inverse, in a classical limit, $\hbar^{(n)}=\hbar / k^{n} \rightarrow 0$. The spectrum and eigenstates of $\hat{U}, \hat{U}^{\prime}, \ldots, \hat{U}^{(n-1)}$ for all $k$ BCs and $n>1$ can be fully reproduced from those of $\hat{U}^{(n)}$ at 
fixed BCs corresponding to either strict periodicity for $k p$ even or antiperiodicity for $k p$ odd. As a first application of all this scheme, we show that some results at fixed BCs can be derived and understood in a simple and transparent way in the general-BCs framework. In particular, it is easy to show in this framework that the eigenstates of $\hat{U}^{(n)}$ at the fixed BCs are all "crystalline". Unlike general crystalline eigenstates of the quantum cat maps [14], the crystal features persist for arbitrarily large perturbations.

We denote by $\mathbf{z}=(u, v)$ the phase-space variables, $[\hat{u}, \hat{v}]=i \hbar$, and we assume that the classical dynamics can be reduced to a $2 \pi \times 2 \pi$ torus $T^{2}$, where it is described by an Anosov map $M$. In general, a smooth map $M$ on $T^{2}$ can be written as $\mathbf{z} \rightarrow M(\mathbf{z})=\bar{M}(\mathbf{z}) \bmod 2 \pi$, where the "lifted" map $\bar{M}$, defined on the entire phase plane $(u, v)$, can be expressed uniquely as the composition of two maps, $\bar{M}=M_{A} \circ M_{1}$ [15]. Here $M_{A}$ is a linear map, $M_{A}(\mathbf{z})=A \cdot \mathbf{z}$, where $A$ is a $2 \times 2$ integer matrix with $\operatorname{det}(A)=1$; by "Anosov" we just mean that $|\operatorname{Tr}(A)|>2$, a condition generically satisfied by $A$. The map $M_{1}$ is defined by $M_{1}(\mathbf{z})=\mathbf{z}+\mathbf{F}(\mathbf{z})$, where $\mathbf{F}(\mathbf{z})$ is a $2 \pi$-periodic vector function of $\mathbf{z}$. For $\mathbf{F}(\mathbf{z})=\mathbf{0}, M$ is a "cat map". The quantization of $\bar{M}$ is the unitary operator $\hat{U}=\hat{U}_{A} \hat{U}_{1}$, where $\hat{U}_{A}$ and $\hat{U}_{1}$ are the quantizations of $M_{A}$ and $M_{1}$, respectively [15]. In the $u$ representation,

$$
\left\langle u_{2}\left|\hat{U}_{A}\right| u_{1}\right\rangle_{\hbar}=\left(\frac{1}{2 \pi i \hbar A_{1,2}}\right)^{1 / 2} \exp \left[\frac{i}{2 \hbar A_{1,2}}\left(A_{1,1} u_{1}^{2}-2 u_{1} u_{2}+A_{2,2} u_{2}^{2}\right)\right] .
$$

We shall assume that $M_{1}$ is the map for a Hamiltonian which is periodic in phase space with unit cell $T^{2}$. As shown in Ref. [15], this is the case if and only if $\int_{T^{2}} \mathbf{F}(\mathbf{z}) d \mathbf{z}=\mathbf{0}$. Then $\hat{U}_{1}$ is the one-step evolution operator for the Weyl quantization of this Hamiltonian and is a periodic operator function $\hat{U}_{1}(\hat{\mathbf{z}} ; \hbar)$, representable by a well defined Fourier expansion. Quantization on a torus requires now that $\hbar=2 \pi / p, p$ integer. The QAM quantizing $M$ is $\hat{U}$ restricted to act on the simultaneous eigenstates of the commuting phase-space translations on $T^{2}, \hat{D}_{1}=\exp (i p \hat{u})$ and $\hat{D}_{2}=\exp (-i p \hat{v})$; the corresponding eigenvalues are $\exp \left(i p w_{1}\right)$ and $\exp \left(-i p w_{2}\right)$, where $\left(w_{1}, w_{2}\right)=\mathbf{w}$ is the Bloch wave vector specifying the toral BCs [2]. An eigenstate $\Psi_{\mathbf{w}}$ of $\hat{D}_{1}$ and $\hat{D}_{2}$ can be an eigenstate of $\hat{U}$ only for those values of $\mathbf{w}$ in the 
Brillouin zone (BZ: $\left.0 \leq w_{1}, w_{2}<2 \pi / p\right)$ satisfying the equation 10 15

$$
A \cdot \mathbf{w}=\mathbf{w}+\pi \mathbf{y} \bmod 2 \pi / p,
$$

where $\mathbf{y} \equiv\left(A_{1,1} A_{1,2}, A_{2,1} A_{2,2}\right)$. We write the general solution of Eq. (2) as follows:

$$
\mathbf{w}=(2 \pi / p) B \cdot\left(\mathbf{r}+p E^{-1} \cdot \mathbf{y} / 2\right) \bmod 2 \pi / p,
$$

where $B=(A-I)^{-1} E, I$ is the identity matrix, $E$ is an arbitrary integer matrix with $\operatorname{det}(E)= \pm 1$, and $\mathbf{r}$ is an integer vector labeling the solutions. There are precisely $k=$ $\left|\operatorname{det}\left(B^{-1}\right)\right|=|2-\operatorname{Tr}(A)|$ distinct vectors (3), as the number of fixed points of $M_{A} \bmod 2 \pi$ [9], forming a lattice in the BZ. We denote by $\mathcal{S}$ the space of states $\Psi_{\mathrm{w}}$ for all these $k$ values of $\mathbf{w}$. The subspace $\mathcal{S}_{\mathbf{w}}$ of $\mathcal{S}$ with a fixed value of $\mathbf{w}$ is $p$-dimensional, i.e., it is spanned by a basis of $p$ independent states [2, [4, whose general expression in the $u$ representation is [19]

$$
\Psi_{b, \mathbf{w}}(u)=\sum_{m=0}^{p-1} \phi_{b}(m ; \mathbf{w}) \sum_{l=-\infty}^{\infty} e^{i l p w_{2}} \delta\left(u-w_{1}-2 \pi m / p-2 \pi l\right),
$$

where $b=1, \ldots, p$. Such a basis is formed, naturally, by the $p$ eigenstates of $\hat{U}$ at fixed $\mathbf{w}$.

We now introduce the torus $T_{B}^{2}$, defined by the vectors $\mathbf{R}_{j}=2 \pi k\left(B_{1, j}, B_{2, j}\right), j=1,2$; $k B$ has integer entries and $T_{B}^{2}$ contains precisely $k$ tori $T^{2}$. Since $B^{-1} A B=E^{-1} A E$ is an integer matrix, the superlattice with unit cell $T_{B}^{2}$ is invariant under $A$, so that the map $\bar{M}$ modulo $T_{B}^{2}$, denoted by $M^{(B)}$, is well defined. To continue, we shall first work out in detail the reflection-hyperbolic case of $\operatorname{Tr}(A)<-2$, choosing $E=I$, so that $[A, B]=0$ and $\operatorname{det}(B)>0$. We shall then specify the changes to be made in the ordinary-hyperbolic case of $\operatorname{Tr}(A)>2$. Let us perform the linear transformation of variables

$$
\mathbf{z}=k B \cdot \mathbf{z}^{\prime}=\sqrt{k} C \cdot \mathbf{z}^{\prime}
$$

where $C=\sqrt{k} B$ and $\mathbf{z}^{\prime}=\left(u^{\prime}, v^{\prime}\right)$. Eq. (5) is the combination of a linear canonical transformation $[\operatorname{det}(C)=+1$, since $\operatorname{det}(B)>0]$ with a scaling by a factor $\sqrt{k}$. Using $[A, B]=0$, it is easy to check that the map $M^{(B)}$ above is transformed by (5) into a map $M^{\prime}$ on $T^{2}$ in 
the $\mathbf{z}^{\prime}$ variables, $M^{\prime}\left(\mathbf{z}^{\prime}\right)=\bar{M}^{\prime}\left(\mathbf{z}^{\prime}\right) \bmod 2 \pi$, with $\bar{M}^{\prime}=M_{A}^{\prime} \circ M_{1}^{\prime}$. Here $M_{A}^{\prime}\left(\mathbf{z}^{\prime}\right)=A \cdot \mathbf{z}^{\prime}$ and $M_{1}^{\prime}\left(\mathbf{z}^{\prime}\right)=(k B)^{-1} \cdot M_{1}\left(\mathbf{z}=k B \cdot \mathbf{z}^{\prime}\right)$. The renormalization transformation $\mathcal{R}_{c}$ in the classical case is then defined by $M^{\prime}\left(\mathbf{z}^{\prime}\right)=\mathcal{R}_{c}[M(\mathbf{z})]$ [20]. Clearly, the cat maps, with $\mathbf{F}(\mathbf{z})=\mathbf{0}$, are fixed points of $\mathcal{R}_{c}$, i.e., $M^{\prime}\left(\mathbf{z}^{\prime}=\mathbf{z}\right)=M(\mathbf{z})$.

The quantum version of (5) implies that $\left[\hat{u}^{\prime}, \hat{v}^{\prime}\right]=i \hbar^{\prime}$, where $\hbar^{\prime}=\hbar / k=2 \pi / p^{\prime}, p^{\prime} \equiv k p$. The quantization $\hat{U}^{\prime}$ of $M^{\prime}\left(\mathbf{z}^{\prime}\right)$ is simply $\hat{U}$ expressed in terms of $\left(\hat{\mathbf{z}}^{\prime}, \hbar^{\prime}\right)$ and acting on the space of the simultaneous eigenstates $\Psi_{\mathbf{w}^{\prime}}^{\prime}$ of the phase-space translations on $T^{2}$ in the $\mathbf{z}^{\prime}$ variables, $\hat{D}_{1}^{\prime}=\exp \left(i p^{\prime} \hat{u}^{\prime}\right)$ and $\hat{D}_{2}^{\prime}=\exp \left(-i p^{\prime} \hat{v}^{\prime}\right)$. It is easy to show that

$$
\hat{D}_{j+1}^{\prime}=\hat{D}\left(\mathbf{R}_{j}\right)=(-1)^{p k^{2} B_{1, j} B_{2, j}} \hat{D}_{1}^{k B_{2, j}} \hat{D}_{2}^{k B_{1, j}}
$$

$\left(j=1,2, \hat{D}_{3}^{\prime} \equiv \hat{D}_{1}^{\prime}\right)$, where $\hat{D}\left(\mathbf{R}_{j}\right)$ are precisely the Weyl-Heisenberg phase-space translations on $T_{B}^{2}$. By expressing $\hat{U}=\hat{U}_{A} \hat{U}_{1}$ in terms of $\left(\hat{\mathbf{z}}^{\prime}, \hbar^{\prime}\right)$, using also the theory of linear quantum canonical transformations [21], we obtain the expected result $\hat{U}^{\prime}=\hat{U}_{A}^{\prime} \hat{U}_{1}^{\prime}$. Here the $u^{\prime}$ representation of $\hat{U}_{A}^{\prime}$ is given by (1) with $u$ and $\hbar$ replaced by $u^{\prime}$ and $\hbar^{\prime}$, respectively, and $\hat{U}_{1}^{\prime}$ is the operator function $\hat{U}_{1}^{\prime}\left(\hat{\mathbf{z}}^{\prime} ; \hbar^{\prime}\right)=\hat{U}_{1}\left(\hat{\mathbf{z}}=k B \cdot \hat{\mathbf{z}}^{\prime} ; \hbar=k \hbar^{\prime}\right)$ [the function $\hat{U}_{1}(\hat{\mathbf{z}} ; \hbar)$ was defined above]. The renormalization transformation $\mathcal{R}$ is then defined by $\hat{U}^{\prime}=\mathcal{R}(\hat{U})$. The quantum cat maps $\left(\hat{U}=\hat{U}_{A}\right)$ are fixed points of $\mathcal{R}$, i.e., $\left\langle u_{2}^{\prime}=u_{2}\left|\hat{U}^{\prime}\right| u_{1}^{\prime}=u_{1}\right\rangle_{\hbar^{\prime}=\hbar}=\left\langle u_{2}|\hat{U}| u_{1}\right\rangle_{\hbar}$.

The space of states $\Psi_{\mathbf{w}^{\prime}}^{\prime}$ for all the $k$ allowed values of $\mathbf{w}^{\prime}$ will be denoted by $\mathcal{S}^{\prime}$. We now show that $\mathcal{S}$ coincides with the subspace $\mathcal{S}_{\mathbf{w}_{0}^{\prime}}^{\prime}$ of $\mathcal{S}^{\prime}$ associated with a particular value $\mathbf{w}_{0}^{\prime}$. Thus, $\mathcal{S}^{\prime}$ is $k$ times larger than $\mathcal{S}$. To show this, let us apply $\hat{D}_{j}^{\prime}, j=1,2$, on a state $\Psi_{\mathrm{w}}$ of $\mathcal{S}$. Using (3), (6), and the fact that $\hat{D}_{j} \Psi_{\mathbf{w}}=\exp \left[i(-1)^{j+1} p w_{j}\right] \Psi_{\mathbf{w}}, j=1,2$, we obtain

$$
\hat{D}_{j}^{\prime} \Psi_{\mathbf{w}}=(-1)^{p A_{j, j+1}} \Psi_{\mathbf{w}}
$$

$\left(A_{2,3} \equiv A_{2,1}\right)$. Rel. (7) means that all $\Psi_{\mathrm{w}}$ in $\mathcal{S}$ are eigenstates of $\hat{D}_{j}^{\prime}, j=1,2$, associated with the same renormalized Bloch wave vector $\mathbf{w}_{0}^{\prime}$. The latter can assume only four values, depending on the matrix $A$, see Table 1 . 


\begin{tabular}{||c||c||c||}
\hline \hline & $k$ even & $k$ odd \\
\hline \hline$p$ even & $\mathbf{w}_{0}^{\prime}=\mathbf{0}$ & $\mathbf{w}_{0}^{\prime}=\mathbf{0}$ \\
\hline \hline$p$ odd & $\mathbf{w}_{0}^{\prime}=\left(\frac{A_{1,2} \pi}{p^{\prime}}, \frac{A_{2,1} \pi}{p^{\prime}}\right) \bmod \frac{2 \pi}{p^{\prime}}$ & $\mathbf{w}_{0}^{\prime}=\left(\frac{\pi}{p^{\prime}}, \frac{\pi}{p^{\prime}}\right)$ \\
\hline \hline
\end{tabular}

Table 1.

It is easy to show that $\mathbf{w}_{0}^{\prime}$ is indeed an allowed value of $\mathbf{w}^{\prime}$ in all four cases. Thus, $\mathcal{S}_{\mathbf{w}_{0}^{\prime}}^{\prime}$ includes $\mathcal{S}$, but since both $\mathcal{S}_{\mathbf{w}_{0}^{\prime}}^{\prime}$ and $\mathcal{S}$ are $k p$-dimensional, they coincide. This completes the proof. Now, by the definition above of $\hat{U}^{\prime}$, the restriction of $\hat{U}^{\prime}$ to $\mathcal{S}_{\mathbf{w}_{0}^{\prime}}^{\prime}=\mathcal{S}$ is just $\hat{U}$. The $k p$ eigenstates of $\hat{U}$ for all $k$ BCs are then precisely the $p^{\prime}$ eigenstates of $\hat{U}^{\prime}$ associated with the value of $\mathbf{w}_{0}^{\prime}$ in Table 1 . When referred to $\hat{U}^{\prime}$, however, these eigenstates should be expressed in a representation based on the operator $\hat{\mathbf{z}}^{\prime}$. If the $k p$ eigenstates of $\hat{U}$ are $\Psi_{b, \mathbf{w}}(u)$ in the $u$ representation, see (四), their $u^{\prime}$ representation will be obtained by applying to $\Psi_{b, \mathrm{w}}(u)$ the unitary transformation corresponding to a linear canonical transformation [21] with matrix $C$, after scaling $u^{\prime}$ by a factor $\sqrt{k}$. The eigenstates of $\hat{U}^{\prime}$ for $\mathbf{w}^{\prime}=\mathbf{w}_{0}^{\prime}$ are thus given by

$$
\Psi_{b^{\prime}, \mathbf{w}_{0}^{\prime}}^{\prime}\left(u^{\prime}\right)=\left(\frac{p}{4 \pi^{2} B_{1,2}}\right)^{1 / 2} \int_{-\infty}^{\infty} d u \exp \left[\frac{-i p}{4 \pi B_{1,2}}\left(k B_{1,1} u^{\prime 2}-2 u^{\prime} u+B_{2,2} u^{2}\right)\right] \Psi_{b, \mathbf{w}}(u),
$$

where $b^{\prime}=b^{\prime}(b, \mathbf{w})$ takes precisely all its $p^{\prime}$ values when $b$ and $\mathbf{w}$ take all their $p$ and $k$ values, respectively; conversely, $\Psi_{b, \mathbf{w}}(u)$ can be fully reproduced from $\Psi_{b^{\prime}, \mathbf{w}_{0}^{\prime}}^{\prime}\left(u^{\prime}\right)$ by inverting Rel. (8) and determining $\mathbf{w}$ by applying $\hat{D}_{1}$ and $\hat{D}_{2}$ on $\Psi_{b, \mathbf{w}}(u)$. If the quasienergies of $\hat{U}$ are $\omega_{b}(\mathbf{w})$, those of $\hat{U}^{\prime}$ for $\mathbf{w}^{\prime}=\mathbf{w}_{0}^{\prime}$ are $\omega_{b^{\prime}(b, \mathbf{w})}^{\prime}\left(\mathbf{w}_{0}^{\prime}\right)=\omega_{b}(\mathbf{w})$. The latter relation and Rel. (8) show the equivalence between the quasienergy eigenvalue problem for $\hat{U}$ for all $k$ BCs and that for $\hat{U}^{\prime}$ at the fixed BCs given by $\mathbf{w}^{\prime}=\mathbf{w}_{0}^{\prime}$.

The case of $\operatorname{Tr}(A)>2$ can be treated similarly only if the integer matrix $E$ in $B=(A-I)^{-1} E$ can be chosen so that $[A, E]=0$ and $\operatorname{det}(E)=-1$. Then one has again $[A, B]=0$ and $\operatorname{det}(C)=+1$ in (5), leading to the same results as above. If $A=K^{2 l}$, where $K$ is any integer matrix with $\operatorname{det}(K)=-1$ and $l$ is an integer, one can choose $E=K$. 
In general, an integer matrix $E$ having the properties above does not exist, and we then make the simple choice $E_{1,1}=-E_{2,2}=1, E_{1,2}=E_{2,1}=0$, corresponding to time reversal. As a result, in all the expressions and equations involving the renormalized quantities, including in Table $1, A$ is replaced by $A^{\prime}=E^{-1} A E$. Thus, the quantum cat maps are now fixed points of $\mathcal{R}$ accompanied by time reversal: Given the eigenstates $\Psi_{b, \mathbf{w}}(u)$ and quasienergies $\omega_{b}(\mathbf{w})$ of $\hat{U}_{A}$ for all BCs, its eigenstates and quasienergies for $\hbar^{\prime}=2 \pi / p^{\prime}$ and $\mathbf{w}^{\prime}=\mathbf{w}_{0}^{\prime}$ are, respectively, $\Psi_{b^{\prime}, \mathbf{w}_{0}^{\prime}}^{\prime *}\left(u^{\prime}\right)$ and $\omega_{b^{\prime}(b, \mathbf{w})}^{\prime}\left(\mathbf{w}_{0}^{\prime}\right)=-\omega_{b}(\mathbf{w})$, where $\Psi_{b^{\prime}, \mathbf{w}_{0}^{\prime}}^{\prime}\left(u^{\prime}\right)$ is given by (8). Time-reversal symmetry $\left(A^{\prime}=A^{-1}\right)$ allows to choose $\Psi_{b^{\prime}, \mathbf{w}_{0}^{\prime}}^{\prime}\left(u^{\prime}\right)$ as real, $\Psi_{b^{\prime}, \mathbf{w}_{0}^{\prime}}^{\prime *}\left(u^{\prime}\right)=\Psi_{b^{\prime}, \mathbf{w}_{0}^{\prime}}^{\prime}\left(u^{\prime}\right)$.

By iterating $\mathcal{R}$, one obtains a sequence of QAMs $\hat{U}^{(n)}=\mathcal{R}^{n}(\hat{U})$ on $T^{2}$, associated with the Planck's constants $\hbar^{(n)}=2 \pi / p^{(n)}, p^{(n)} \equiv k^{n} p$. General $\hat{U}^{(n)}=\hat{U}_{A}^{(n)} \hat{U}_{1}^{(n)}$ represent perturbations of the quantum cat map $\hat{U}_{A}$, or its time inverse $\hat{U}_{A^{\prime}}$ (only for $n$ odd), in a classical limit, $n \rightarrow \infty$. The perturbation $\hat{U}_{1}^{(n)}\left(\hat{\mathbf{z}}^{(n)} ; \hbar^{(n)}\right)$ is periodic in $\hat{\mathbf{z}}^{(n)}$ with a unit cell $T_{n}^{2}=(k B)^{-n} \cdot T^{2}$, which is $k^{n}$ times smaller than $T^{2}$. In the generalization of Table 1 to $n>1, p^{(n-1)}=k^{n-1} p$ is always even when $k$ is even, so that $\mathbf{w}_{0}^{(n)}$ can take only two values: $\mathbf{w}_{0}^{(n)}=\mathbf{0}$ (for $k p$ even) and $\mathbf{w}_{0}^{(n)}=\left(\pi / p^{(n)}, \pi / p^{(n)}\right)$ (for $k p$ odd), corresponding to strictly periodic and antiperiodic BCs, respectively. The eigenstates of $\hat{U}^{(n)}$ for $\mathbf{w}^{(n)}=\mathbf{w}_{0}^{(n)}$ are connected with those of $\hat{U}^{(n-1)}$ for all $k$ BCs by a relation analogous to Rel. (8). The quasienergies are related by $\omega_{b^{(n)}}^{(n)}\left(\mathbf{w}_{0}^{(n)}\right)=\omega_{b^{(n-1)}}^{(n-1)}\left(\mathbf{w}^{(n-1)}\right)$. Thus, the spectrum and eigenstates of $\hat{U}, \ldots, \hat{U}^{(n-1)}$ for all BCs can be fully reproduced from those of $\hat{U}^{(n)}$ for $\mathbf{w}^{(n)}=\mathbf{w}_{0}^{(n)}$.

We now show that all the eigenstates of $\hat{U}^{(n)}$ for $\mathbf{w}^{(n)}=\mathbf{w}_{0}^{(n)}$ are "crystalline". By construction, (8) are eigenstates of $\hat{D}_{j}=\hat{D}^{\prime}\left(\mathbf{R}_{j}^{\prime}\right), j=1,2$, where $\hat{D}^{\prime}\left(\mathbf{R}_{j}^{\prime}\right)$ are WeylHeisenberg translations in the $\left(u^{\prime}, v^{\prime}\right)$ phase space by vectors $\mathbf{R}_{j}^{\prime}$ defining precisely the unit cell $T_{1}^{2}=(k B)^{-1} \cdot T^{2}$, which is $k$ times smaller than $T^{2}$. Thus, the Husimi density of all the eigenstates of $\hat{U}^{\prime}$ for $\mathbf{w}^{\prime}=\mathbf{w}_{0}^{\prime}$ is exactly periodic on a lattice with unit cell $T_{1}^{2}$. The eigenstates are then crystalline [14]: each cell contains $p$ of the $k p$ Husimi zeros in $T^{2}$. For $n>1$ and $\mathbf{w}^{(n)}=\mathbf{w}_{0}^{(n)}$, the $k^{n} p$ eigenstates of $\hat{U}^{(n)}$ can be grouped into $n$ sets: the $l$ th 
set, $l=1, \ldots, n$, consists of $k^{l-1} p\left(k+\delta_{l, 1}-1\right)$ crystalline eigenstates with unit cell $T_{n-l+1}^{2}$ and $k^{l-1} p$ Husimi zeros in each cell. Unlike general crystalline eigenstates of the quantum cat maps [14], the crystal features of all this complete basis of eigenstates persist under an arbitrarily large perturbation $\hat{U}_{1}^{(n)}$ in $\hat{U}^{(n)}$.

Another interesting result at fixed BCs concerns the expansion (44) for the eigenstates (8) and can be easily derived in the case when the vectors (3) form a square lattice in the BZ, $\mathbf{w}=2 \pi\left(r_{1}, r_{2}\right) /(g p), r_{1}, r_{2}=0, \ldots, g-1, g$ integer (see the conditions for this in note [22]). Since $B=I / g$ [22], the transformation (5) is simply $\mathbf{z}=g \mathbf{z}^{\prime}$, and the eigenstates $\Psi_{b^{\prime}, \mathbf{w}_{0}^{\prime}}^{\prime}\left(u^{\prime}\right)$ of $\hat{U}^{\prime}$ can be easily determined, without using (8), by just substituting $u=g u^{\prime}$ in (伍). After rearranging terms, we find that $\Psi_{b^{\prime}, \mathbf{w}_{0}^{\prime}}^{\prime}\left(u^{\prime}\right)$ is given by the expression in Eq. (4) with all the quantities replaced by their primed counterparts and $\mathbf{w}^{\prime}=\mathbf{w}_{0}^{\prime}=\mathbf{0}$. For given $b$ and $\mathbf{w}$, an expansion coefficient $\phi_{b^{\prime}(b, \mathbf{w})}\left(m^{\prime} ; \mathbf{w}_{0}^{\prime}\right), m^{\prime}=0, \ldots, p^{\prime}-1$, is nonzero only if there exists an integer pair $(m, l), m=0, \ldots, p-1, l=0, \ldots, g-1$, solving the Diophantine equation $p g l+g m+r_{1}=m^{\prime}$. The solution is then unique and $\phi_{b^{\prime}}\left(m^{\prime} ; \mathbf{w}_{0}^{\prime}\right)=\exp \left(2 \pi i l r_{2} / g\right) \phi_{b}(m ; \mathbf{w})$, associated with a sparse expansion. In particular, for $p=1$, i.e., $\hbar^{\prime}=2 \pi / g^{2}$, we can choose $\phi_{b}(m ; \mathbf{w})=1$, and the only nonzero coefficients are $\phi_{b^{\prime}}\left(m^{\prime} ; \mathbf{w}_{0}^{\prime}\right)=\exp \left(2 \pi i l r_{2} / g\right)$ with $m^{\prime}=g l+r_{1}$. This result can be obtained directly by applying the methods in Refs. 10,14, where the general case of $2 \pi / \hbar=$ perfect square was studied in detail.

In conclusion, we have shown that the quasienergy problem of general QAMs for all BCs can be exactly reduced to a fixed-BCs problem. This reduction is possible due to a distinctive feature of QAMs: For given $\hbar=2 \pi / p$, the $k$ allowed Bloch wave vectors $\mathbf{w}$ form a lattice in the $\mathrm{BZ}$, completely determined by the associated matrix $A$. Thus, this reduction is not possible for the special torus maps with $\operatorname{Tr}(A)=2$, e.g., the kicked rotor and the kicked Harper maps, since the number of BCs is infinite for them. The reduction is implemented by a renormalization transformation $\mathcal{R}$ exhibiting nontrivial features: For $\operatorname{Tr}(A)<-2$, the quantum cat maps are always fixed points of $\mathcal{R}$, while for $\operatorname{Tr}(A)>2$ they are generally fixed 
points only of $\mathcal{R}$ accompanied by time reversal. These features reflect the scaling invariance of the quantum kernel (1). As a first application, we have shown that some results at fixed BCs can be derived and understood in a simple and transparent way in the general-BCs framework. In particular, one can easily establish the existence of a complete fixed-BCs basis of crystalline eigenstates in a classical limit for arbitrarily large perturbations. The results in this Letter may be applied to study several aspects of the general-BCs problem for QAMs using known results and methods at fixed BCs. An important and unexplored issue is the sensitivity of eigenstates to variations in the BCs and its relation to classical phase-space structures, especially in a regime of mixed phase space (large perturbations).

\section{Acknowledgments}

The author would like to thank S. Nonnenmacher for helpful comments and correspondence. Comments from J.P. Keating and Z. Rudnick are gratefully acknowledged. This work was partially supported by the Israel Science Foundation administered by the Israel Academy of Sciences and Humanities. 


\section{REFERENCES}

[1] P. Leboeuf, J. Kurchan, M. Feingold, and D.P. Arovas, Phys. Rev. Lett. 65, 3076 (1990); Chaos 2, 125 (1992).

[2] I. Dana, Phys. Rev. E 52, 466 (1995).

[3] I. Dana, M. Feingold, and M. Wilkinson, Phys. Rev. Lett. 81, 3124 (1998).

[4] I. Dana, Y. Rutman, and M. Feingold, Phys. Rev. E 58, 5655 (1998).

[5] V.I. Arnold, Geometrical Methods in the Theory of Ordinary Differential Equations (Springer-Verlag, New York, 1988).

[6] J.H. Hannay and M.V. Berry, Physica (Amsterdam) 1D, 267 (1980).

[7] I.C. Percival and F. Vivaldi, Physica (Amsterdam) 25D, 105 (1987).

[8] I. Dana, Physica (Amsterdam) 39D, 205 (1989).

[9] J.P. Keating, Nonlinearity 4, 277, 309 (1991).

[10] S. Knabe, J. Phys. A 23, 2023 (1990).

[11] M. Degli Esposti, Ann. Inst. Henri Poincaré 58, 323 (1993).

[12] M. Degli Esposti, S. Graffi, and S. Isola, Commun. Math. Phys. 167, 471 (1995).

[13] A. Bouzouina and S. De Bièvre, Commun. Math. Phys. 178, 83 (1996).

[14] S. Nonnenmacher, Nonlinearity 10, 1569 (1997).

[15] J.P. Keating, F. Mezzadri, and J.M. Robbins, Nonlinearity 12, 579 (1999).

[16] M. Basilio de Matos and A.M. Ozorio de Almeida, Ann. Phys. 237, 46 (1995).

[17] T.O. de Carvalho, J.P. Keating, and J.M. Robbins, J. Phys. A 31, 5631 (1998).

[18] M.V. Berry, J.P. Keating, and S.D. Prado, J. Phys. A 31, L245 (1998). 
[19] This is derived from the $v$ representation $\Psi_{\mathbf{w}}(v)$ in Ref. [2].

[20] One has $M^{\prime}=M_{k B}^{-1} \circ M^{(B)} \circ M_{k B}$, where $M_{k B}$ corresponds to a transformation of variables, $\mathbf{z}=k B \cdot \mathbf{z}^{\prime}$. If $M^{(B)}$ is viewed as the result of an operation on $M$, i.e., replacing $T^{2}$ by $T_{B}^{2}, M^{\prime}$ has precisely the form of a renormalized map. See, e.g., R.S. MacKay, Renormalization in Area-Preserving Maps (World Scientific, Singapore, 1993).

[21] M. Moshinski and C. Quesne, J. Math. Phys. 12, 1772 (1971).

[22] This case will occur only if $p \mathbf{y} / 2$ in (3) is an integer vector and the matrix $E$ can be chosen so that $B=(A-I)^{-1} E=I / g$. This implies that $A=I+g E$. It is easy to show that the latter relation is satisfied if and only if $A=s E^{2}$ with $\operatorname{Tr}(E)=s g$, where $s=$ $\operatorname{sgn}[\operatorname{Tr}(A)]=-\operatorname{sgn}[\operatorname{det}(E)]$. One can then easily find matrices $A$ for any $g$. 